\title{
Causes of ano-rectal bleeding
}

\author{
T. DEHN \\ M.B., B.S., F.R.C.S. \\ F. P. McGinN \\ M.Phil., M.S., F.R.C.S.
}

Professorial Surgical Unit, St Bartholomew's Hospital, London, and Surgical Unit, Southampton Genera跑

Hospital, Southampton

\section{Summary}

The causes of ano-rectal bleeding as the only symptom in 110 patients were studied. A diagnosis was obtained in $77 \%$ of patients after clinical examination, sigmoidoscopy and proctoscopy, increasing to $97 \%$ after radiology and colonoscopy. Anal causes of bleeding were found in $81 \%$ of the patients, while a source in the rectum or colon accounted for only $16 \%$.

\section{Introduction}

Ano-rectal bleeding is a common symptom which may be associated with local conditions such as haemorrhoids and fissures but may also be the presenting symptom with neoplasia, diverticular disease or inflammatory disease of the colon or rectum. Many patients with rectal or colonic disease have associated symptoms such as change of bowel habit or loss of weight, and it is uncertain how frequently such conditions present with the single symptom of rectal bleeding.

In order to observe the relative frequency of the conditions that present with ano-rectal bleeding alone, a study was made, over one year, of patients whose sole symptom was rectal bleeding. Each patient was seen at a rectal clinic and all had a full examination, followed by inspection and digital examination of the rectum, sigmoidoscopy and proctoscopy and, when indicated, a barium enema examination. Patients with any other symptom in addition to rectal bleeding were excluded from the study.

\section{Results}

One hundred and ten consecutive patients were seen with the solitary symptom of rectal bleeding. There were 60 men (age range 23-83 years, mean 48 years, s.d. \pm 13.3 years), and 50 women (age range 18-83 years, mean 47 years, s.d. $\pm 18 \cdot 1$ years). The final diagnosis in these patients of the causes of rectal bleeding are shown in Table 1.

\section{Lesions of the anal canal}

Haemorrhoids alone were responsible for the
TABLE 1.

Final diagnosis in 110 patients with ano-rectal bleeding

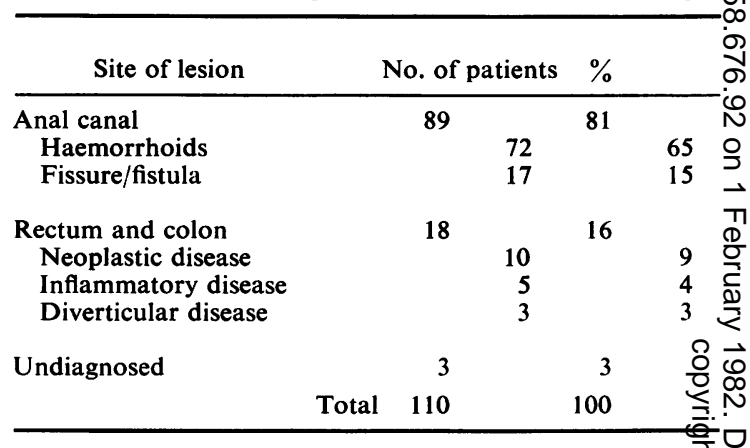

bleeding in 72 patients (46 males and 26 female $\vec{s})$. Anal fissures were present in 16 patients $(5$ men and $\bar{O}$ 11 women) and a fistula in ano was present in one man.

Lesions of the rectum and colon

The diagnoses of patients with bleeding from the colon and rectum are shown in Table 2. Carcinoma

TABLE 2.

Final diagnosis in 18 patients with colo-rectal bleeding

\begin{tabular}{lc}
\hline Neoplastic disease & No. of patients \\
\hline Benign & 5 \\
Malignant & 5 \\
& \\
Inflammatory disease & \\
Proctocolitis & 4 \\
Crohn's disease & 1 \\
Diverticular disease & 3 \\
\hline
\end{tabular}

was present in 5 patients. Rectal carcinomas were $\omega$ present in 3, carcinoma of the sigmoid colon in one, and carcinoma of the transverse colon in another. Benign rectal polyps were found in 5 patients, and all polyps were removed completely through the sig-? moidoscope or at colonoscopy. In addition, $4 \frac{T}{T}$ patients with neoplastic disease also had haemorr- $\frac{\vec{D}}{\mathrm{O}}$ 
hoids ( 3 had rectal polyps and one had a carcinoma of the transverse colon).

Inflammatory disease of the colon and rectum was thought to be responsible for the rectal bleeding in 5 patients; 4 had proctocolitis and one had Crohn's disease. In all 5 patients the bleeding stopped after treatment of the inflammatory bowel disease. Diverticular disease was considered to be the cause of bleeding in 3 patients.

\section{Undiagnosed causes}

In 3 patients the cause of bleeding was not diagnosed. None of the 3 displayed any abnormality on examination, sigmoidoscopy or on barium enema examination. No further bleeding occurred after follow-up review of the patients at 6- and 12-week intervals, and faecal testing for occult blood was negative on each occasion. Colonoscopy examination was not performed on these patients because colonoscopy was reserved for patients with either proved rectal bleeding which persisted after normal sigmoidoscopy, proctoscopy and barium enema examinations, or in those patients where there was doubt of the barium enema result.

\section{Relative value of the various stages in the diagnosis}

Notwithstanding that a good history can give a reasonable indication of the cause of rectal bleeding, examination and palpation enabled a definitive diagnosis to be made in those patients with fissures and local problems $(15 \%$ of the total number studied). Sigmoidoscopy and proctoscopy, however, enable diagnosis to be made of haemorrhoids, most tumours of the rectum and recto-sigmoid, and the majority of inflammatory diseases of the large bowel $(77 \%$ of patients were diagnosed by these procedures in the study).
Barium enema examination (usually double contrast) was performed on 22 patients $(20 \%)$. Seventeen were reported as normal, 3 demonstrated diverticular disease and in 2 a colonic carcinoma was revealed (one a transverse colonic and the other a sigmoid tumour). None of the 5 patients with rectal polyps had any further polyps seen on barium enema.

\section{Comment}

This series is small, since it included patients whose only complaint was that of rectal bleeding. It was undertaken at a busy district hospital and the results are very similar to those of a recent study undertaken at a specialist hospital (Williams and Thomson, 1977).

Benign conditions of the anal canal are by far the most common cause of rectal bleeding, but they can only be confidently diagnosed after sigmoidoscopy and proctoscopy. The barium enema examination was only undertaken after a thorough clinical examination had been performed. Of the 110 patients in this study, $15 \%$ were diagnosed by inspection and palpation, $92 \%$ had been diagnosed at the completion of sigmoidoscopy and proctoscopy, while less than $5 \%$ were diagnosed by barium enema examination. The relatively low diagnostic rate after barium enema examination probably occurred because patients with any other symptom, such as change of bowel habit, were excluded from the study.

\section{References}

Williams, J.T. \& Thomson, J.P.S. (1977) Ano-rectal bleeding: a study of its causes and investigative yields. Practitioner, $219,327$. 